\title{
Timomas. A propósito de un caso clínico
}

\author{
Iván Duhalde Sch, Luis Jiménez V, Daniel Carpio P.
}

\section{RESUMEN}

Los timomas son los tumores más frecuentes del mediastino anterosuperior. Su pronóstico es muy favorable si se diagnostica en etapas I y II. Se presenta el caso de un hombre de 32 años de edad, a quien se le diagnosticó un tumor de mediastino etapa I cuyo resultado anatomopatológico fue un timoma. Se analizan los aspectos relevantes de la patología con énfasis en su clasificación, diagnóstico y tratamiento. (Palabras claves/Key words: Timoma/Tymoma; Tumores mediastínicos/ Mediastinal tumours; Clasificación/Classification; Diagnóstico/Diagnosis; Tratamiento/Treatment).

\section{INTRODUCCIÓN}

Los timomas son uno de los tumores mediastínicos más frecuentes, pudiendo representar hasta el $47 \%$ de todas las masas mediastínicas. Estos tumores se desarrollan a expensas de la trama epitelial del timo ${ }^{1}$. Su diagnóstico se realiza en su mayoría en etapas I y $\|^{2}$, por lo que la evolución es generalmente muy favorable. Además se trata de tumores un tanto especiales, por cuanto muestran tendencia a una invasión local y están asociados a varias enfermedades sistémicas, en particular la miastenia gravis.

El objetivo de esta comunicación es presentar el caso clínico de un enfermo portador de esta inusual patología y comentar algunos de sus particulares aspectos.

\section{CASO CLÍNICO}

Paciente de 32 años, sexo masculino, sin antecedentes patológicos importantes, que desde hace aproximadamente un año, presenta cuadros bronquiales agudos a repetición. En el último episodio se solicita radiografía de tórax que revela masa mediastínica (Figura 1 ). Se realiza tomografía axial computada de tórax que confirma un tumor de mediastino anterosuperior, de $4 \times 5,1 \times 5 \mathrm{~cm}$, con límites bien definidos,

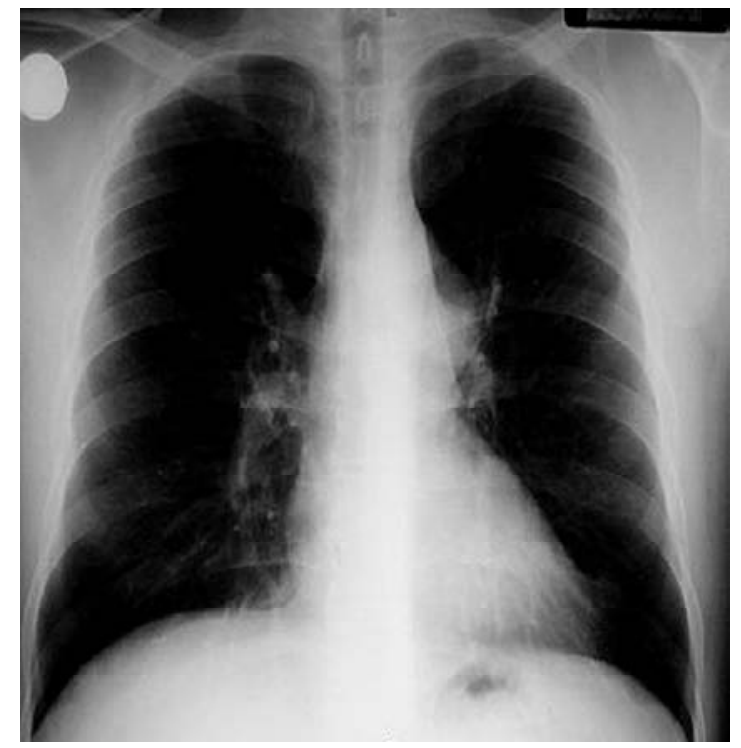

FIGURA 1. Radiografía de tórax que muestra un tumor mediastínico que se insinúa en hemitórax izquierdo.

homogéneo, de crecimiento hacia hemitórax izquierdo (Figura 2). Todos los estudios de laboratorio fueron dentro de límites normales.

Con el diagnóstico de probable linfoma y con fines biópticos, fue intervenido quirúrgicamente el 29 de Mayo de 2002, 


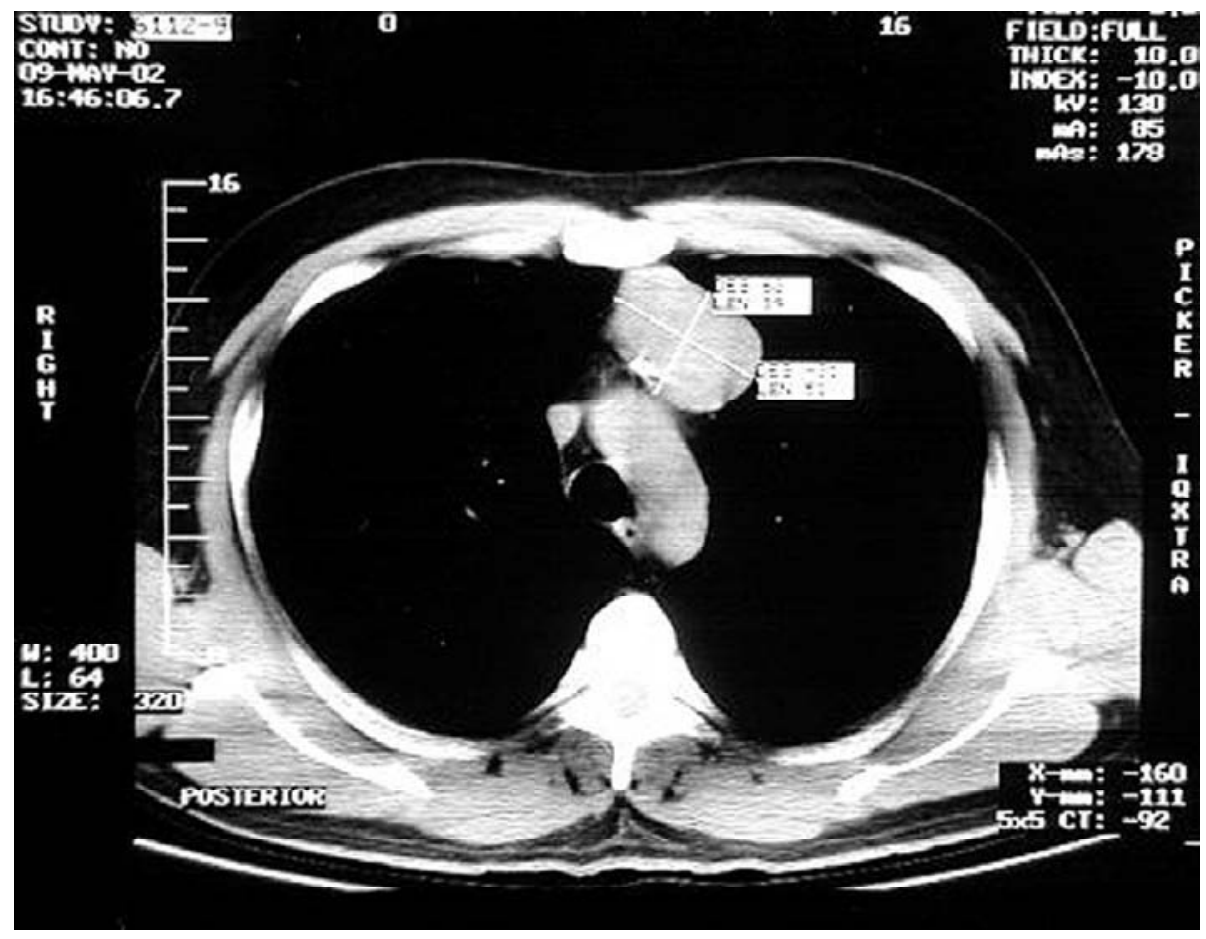

FIGURA 2. Tomografía axial computada que muestra una masa en mediastino anterior de $4 \times 5,1 \times 5 \mathrm{~cm}$, con márgenes bien definidos, homogénea.

inicialmente por videotoracoscopía, encontrando masa enucleable, por lo que se decide convertir y se realiza toracotomía en $5^{\circ}$ espacio intercostal izquierdo, de tamaño mediano. Se extirpa el tumor sin dificultad, ya que no presentaba infiltración a las estructuras vecinas y con un buen plano de clivaje, logrando su resección completa.

Macroscópicamente no se evidenciaban signos de invasión de su cápsula, clasificándolo en etapa I.

La evolución postoperatoria fue satisfactoria. Actualmente el paciente se encuentra en buenas condiciones, con cuadro bronquial de tipo exudativo rebelde al tratamiento habitual.

\section{ANATOMÍA PATOLÓGICA}

La muestra corresponde a un tumor encapsulado y polilobulado que mide $7,5 \times 5,5$ $x 3,5 \mathrm{~cm}$, cuya superficie es de color pardo. Al corte presenta consistencia elástica y coloración pardo blanquecina (Figura 3).

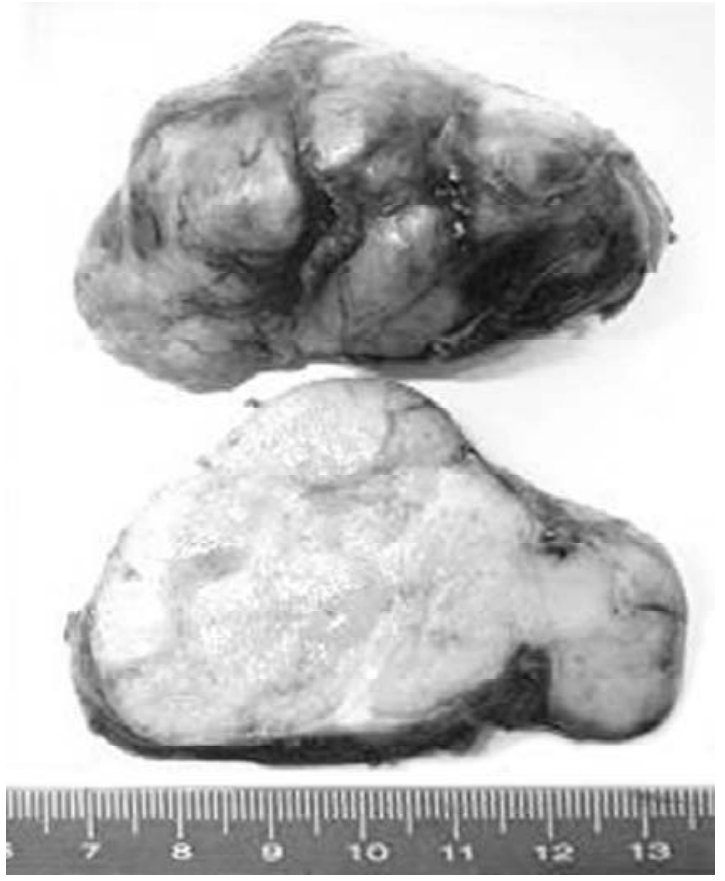

FIGURA 3. Tumor encapsulado, multilobulado. 
Histológicamente se aprecia encapsulado y está compuesto por una mezcla de células de tamaño mediano, de núcleos vesiculosos, sin atipias, y una población de estirpe linfoide, cuyas células son pequeñas, de escaso citoplasma y núcleos monomorfos. Ocasionalmente, las células epitelioideas se observan fusadas (Figura 4).

El estudio con técnicas de inmunohistoquímica revela positividad del citoplasma de las células epitelioideas con cóctel de citokeratinas (Figura 5), positividad de membranas nucleares de las células linfoides con CD-3, para población de linfocitos de estirpe T (Figura 6) y ocasionales células positivas con CD-20 o PAN $B$, especialmente entre los lóbulos tumorales.

\section{COMENTARIO}

Los timomas pueden llegar a representar entre un $26 \%$ a un $50 \%$ de los tumores del mediastino ${ }^{2}$ y un $82,6 \%$ de los tumores del timo.

Histológicamente se reconocen cuatro tipos de tejidos: un tejido predominantemente epitelial, predominantemente linfático, mixto (epiteliales y linfáticos) y fusiforme (predominio epitelial con células fusiformes predominantes).

Desde el punto de vista clínico la clasificación más utilizada es la de Masaoka y cols. (1981), que está basada en el grado de desarrollo del tumor.

\section{Grado Características}

I Encapsulado totalmente, sin objetivarse invasión macro o microscópica de la cápsula.

II Invasión macro y microscópica de la cápsula tumoral y diseminación a la grasa y/o pleura mediastínica.

III Invasión macroscópica de otros tejidos adyacentes como el pericardio, grandes vasos o parénquima pulmonar.

IV a Metástasis pericárdicas y/o pleura mediastínica.

IV b Metástasis linfáticas o hematógenas a distancia.

En el diagnóstico de timoma se debe considerar que éste es un tumor que se

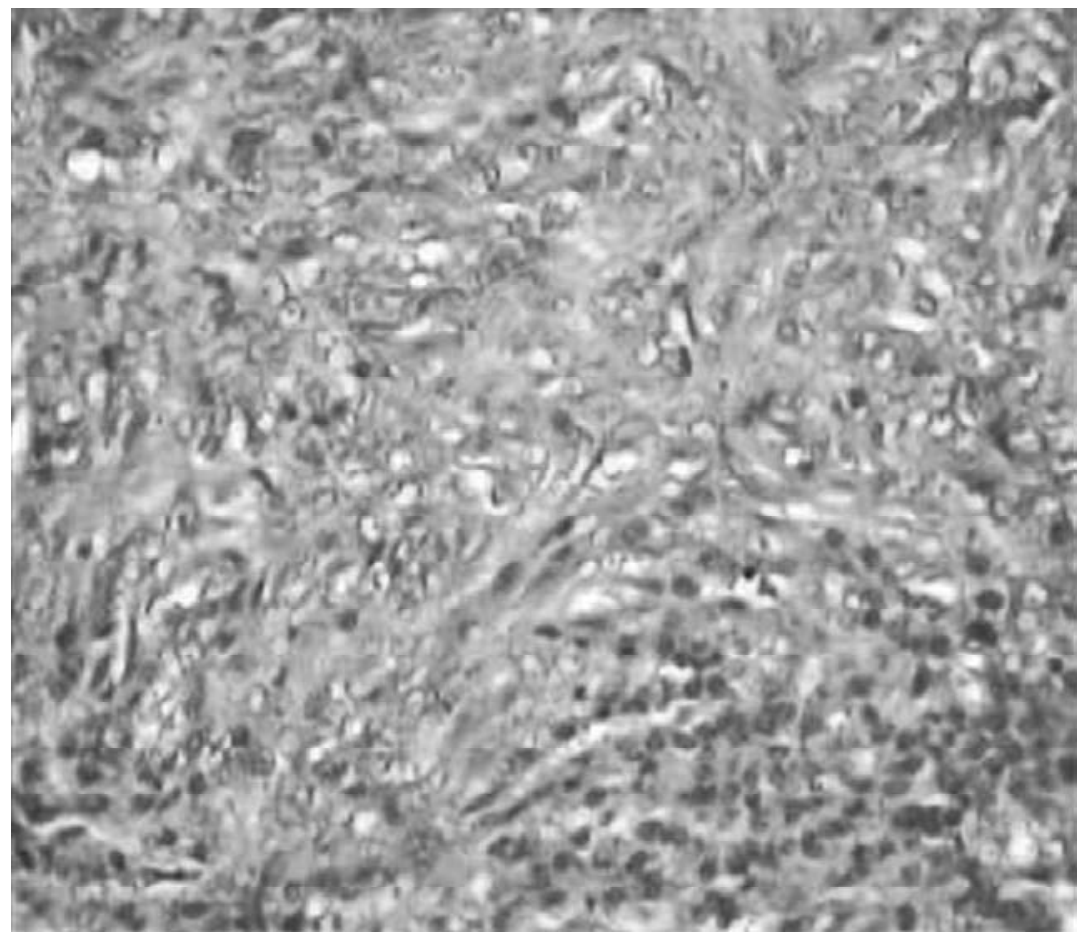

FIGURA 4. Tumor compuesto por una mezcla de células tímicas epitelioideas y linfocitos pequeños. H-E 200x. 


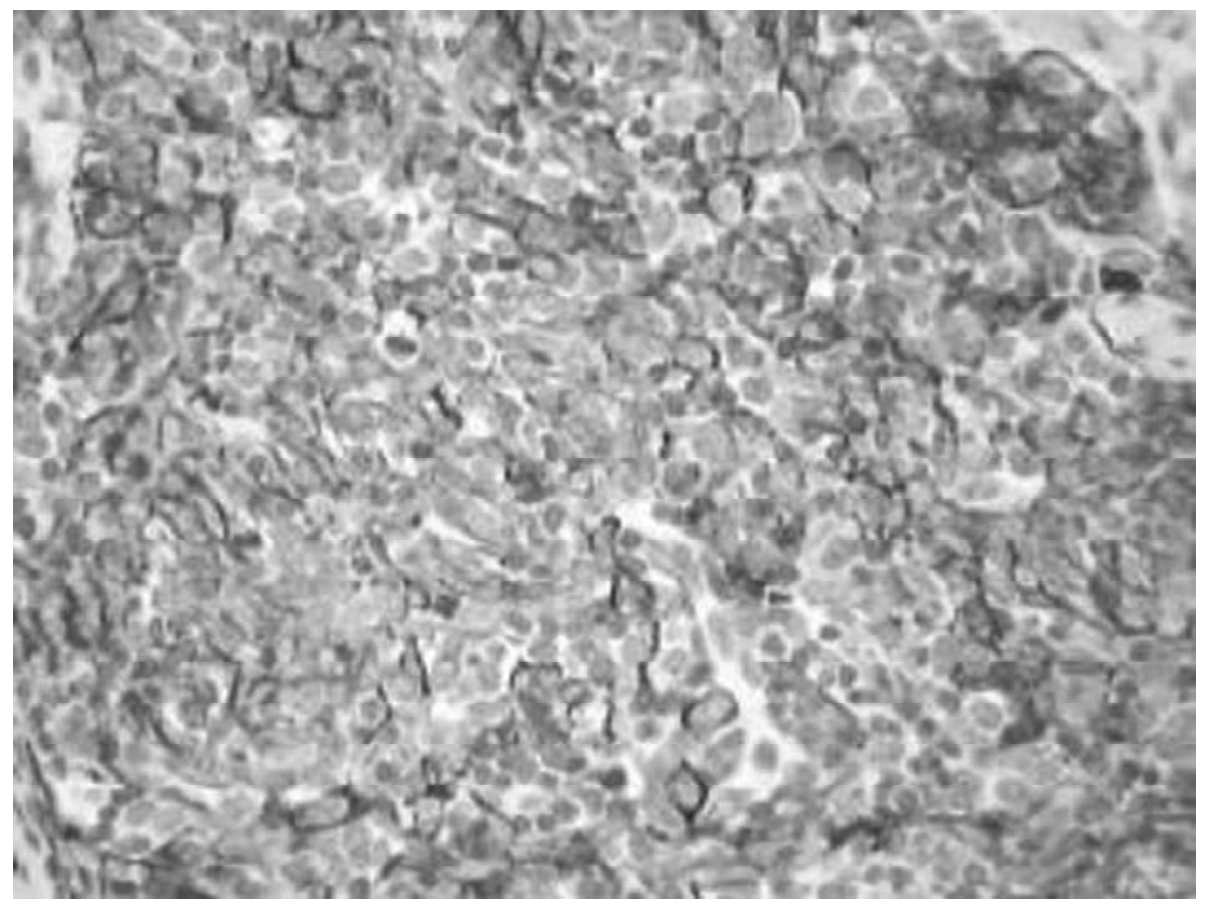

FIGURA 5. Tinción positiva del citoplasma del componente epitelioideo con pancitokeratina. 200x.

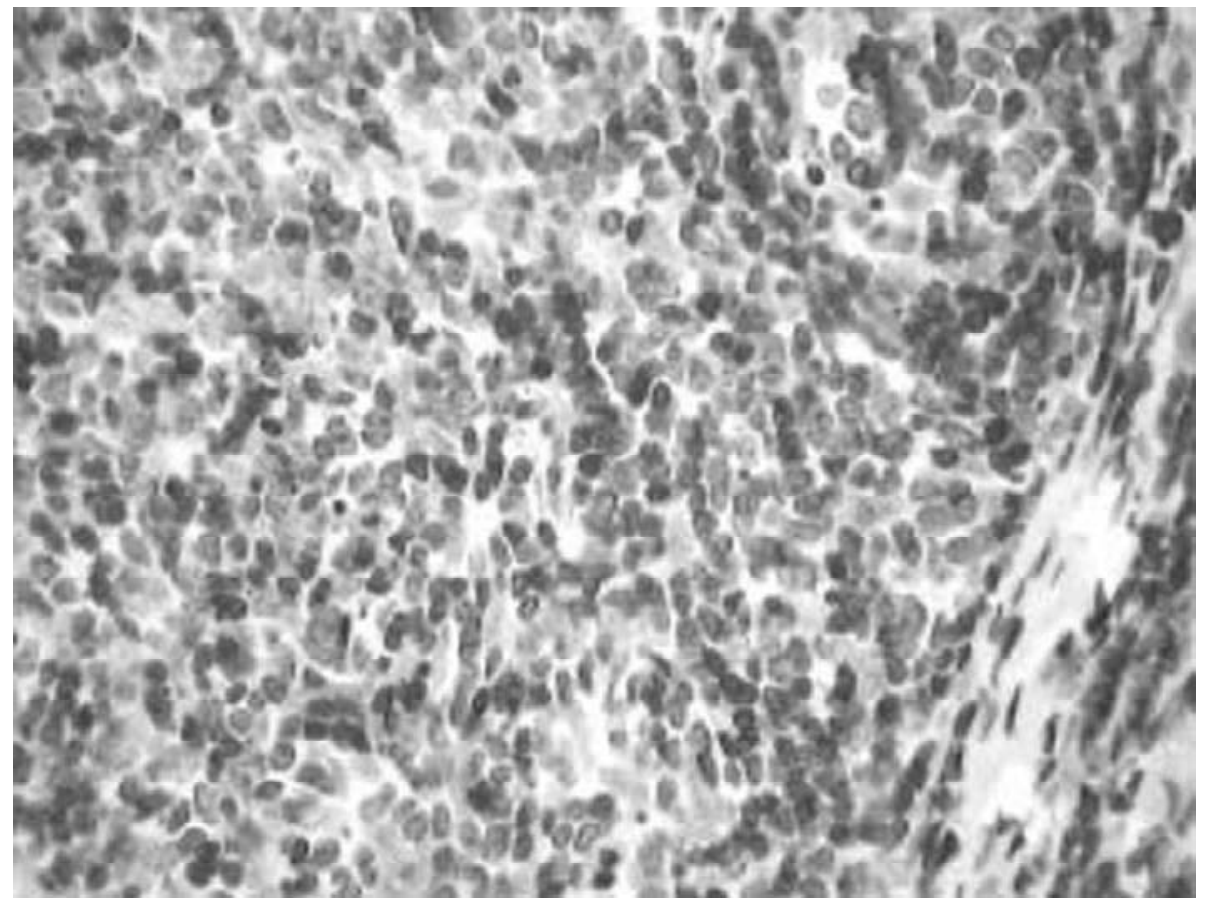

FIGURA 6. Tinción positiva de las membranas celulares del componente linfoide. 200x. 
presenta alrededor de los 55 años de edad ${ }^{2}$ y que afecta, tanto a hombres como mujeres en igual proporción ${ }^{3}$. La clínica es poco específica, y no guarda relación con tamaño del tumor. Los síntomas que con mayor frecuencia se presentan son dolor torácico, tos, opresión torácica, síncope vagal, disnea y taponamiento cardíaco ${ }^{2}$. Además se presentan asociados a patologías, siendo la más relevante la miastenia gravis que se encuentra entre un $21 \%$ a $35 \%{ }^{2}$.

Las manifestaciones radiológicas del timoma pueden variar desde una sutil anormalidad en el contorno mediastínico hasta una amplia masa anterior. En ausencia de invasión pleural, las superficies visceral y parietal de la misma, están desplazadas marginalmente y muestran un borde lobular. Los timomas invasivos pueden tener características radiológicas similares a los de las lesiones encapsuladas. Sin embargo, son más frecuentes manifestaciones de invasión pleural tales como ensanchamiento focal de la pleura, masas pleurales o engrosamiento circunferencial nodular de la pleura que encajonaría completamente los pulmones.

Otros medios de diagnósticos son la TAC (convencional o helicoidal) que permite el estudio de la localización, morfología y extensión de los tumores.

Se cree que la resolución de la TAC helicoidal es superior a la convencional en procesos mediastínicos, aunque no hay trabajos que lo avalen. La resonancia nuclear magnética se utiliza en casos con sospecha de infiltración vascular, pericárdica o miocárdica ${ }^{4}$.

La confirmación histopatológica se puede realizar por medio de punción-aspiración y biopsia por mediastinotomía, con la pieza completa o muestras obtenidas durante el acto quirúrgico.

En el tratamiento de los timomas se debe considerar que son neoplasias de crecimiento relativamente Iento. Cuando se tratan tempranamente ofrecen un excelente pronóstico de supervivencia a largo plazo. La cirugía, la radioterapia y la quimioterapia juegan un papel en el tratamiento.

Cirugía. La extirpación quirúrgica del tumor es considerada como el tratamiento de primera elección. El cirujano deberá evaluar la extensión del tumor e identificar sus propiedades invasivas marcando las áreas sospechosas para ser examinadas por el patólogo. Aunque el cirujano es considerado como el más adecuado para juzgar el grado de invasión del tumor, a menudo es difícil determinar macroscópicamente las áreas invadidas. La completa resección es el mejor predictor de la supervivencia a largo plazo. La esternotomía media es el procedimiento más frecuentemente empleado, ya que da acceso a todo el mediastino y a ambos espacios pleurales. La toracotomía lateral se emplea en los tumores de amplio predominio hacia un hemitórax. El timoma se deberá extirpar con la cápsula completa, no recomendándose la toma de biopsia para evitar la diseminación.

Actualmente se está usando la cirugía videotoracoscópica en el tratamiento del timoma con resultados comparables a la cirugía abierta ${ }^{5}$.

Radioterapia. Los timomas son generalmente sensibles a la radiación, por lo que existe acuerdo sobre el valor de la radioterapia después de la resección de tumores grado II y III. En tumores grado I, la mayoría de los autores estiman que no está indicada la radioterapia. Las dosis recomendadas son de 30 a 60 Gy.

Quimioterapia. En los últimos años la quimioterapia ha ido adquiriendo importancia creciente en el tratamiento de los timomas aunque no se han establecidos directrices definidas. Se han usado como monoterapia la Adriamicina, Cisplatino, Ciclofosfamida y los corticoides en el tratamiento de los timomas invasivos.

La combinación de agentes quimioterápicos suelen dar mejores resultados como Doxorubicina, Cisplatino, Vincristina, que produce una tasa de $92 \%$ de respuesta y un $43 \%$ de remisión 6 . Todavía no está establecido si los regímenes de quimioterapia de combinación son más efectivos que los agentes únicos y aún no existen comparaciones prospectivas.

Estudios han cifrado las recurrencias de los timomas entre un $11 \%$ y $20 \%^{7}$ en los tumores invasivos y entre un $1 \%$ y un $5 \%$ en los no invasivos. La resección de los casos de recidiva local es el tratamiento de elección.

La supervivencia está determinada por la estadificación clínica en función del grado de invasión. Así, Káiser ${ }^{8}$ presenta una sobrevida a 10 años del $85 \%$ de los tumores encapsulados $y$ del $52 \%$ de los invasores. 


\section{RERERENCIAS}

1. Wilkins EW. Thymoma. In: Pearson FG (ed), Thoracic Surgery. New York, Churchill Livingstone Inc,1995; 1419-25

2. Cañizares M A, Arnau A, Alberola A, Pérez A, Hostalet F, Monsalvá E, et al: Timomas. Estudios retrospectivos. Arch Bronconeumol 1999; 35: 324-8

3. Regnard J F, Magdeleinat P, Dromer C, Dulmet $\mathrm{E}$, Montpreville V, Levi JF, et al: Prognostic factors and long term results after thymoma resection: a series of 307 patients. J Thorac Cardiovasc Surg 1996; 112: 376-84

4. Rea F, Sartori F, Loy M, Calabro F, Fornasiero $A$, Daniele $\mathrm{O}$, et al: Chemotherapy and operation for invasive thymoma. J Thorac Cardiovasc Surg 1993; 106: 543-9
5. Yim A P: Video-assisted thoracoscopic resection of anterior mediastinal masses. Int Surg 1996; 81: 350-3

6. Fornasiero A, Daniele O, Ghiotto C, Piazza M, Fiore-Donati L, Calabro F, et al: Chemotherapy for invasive thymoma. A 13-year experience. Cancer 1991; 68: 30-3

7. Ruffini E, Mancuso M, Oliaro A, Casadio C, Cavallo A, Cianci, et al: Recurrence of thymoma: analysis of clinicopathologic features, treatment and outcome. J Thorac Cardiovasc Surg 1997: 113: $55-63$

8. KaiserL R, Martini N: Clinical management of thymoma: the Memorial Sloan-Kettering Cancer Center experience. New York: Strends Cap 15: 176-81. 